\title{
Clinical and Histopathological Features of Corneal Primary Acquired Melanosis and Melanoma
}

\author{
Aaron Yeung $^{\mathrm{a}}$ Ogul E. Uner ${ }^{\mathrm{b}}$ Jill R. Wells ${ }^{\mathrm{a}}$ Hans E. Grossniklaus ${ }^{\mathrm{a}, \mathrm{c}}$ \\ aDepartment of Ophthalmology, Emory University School of Medicine, Atlanta, GA, USA; bSchool of Medicine, Emory \\ University, Atlanta, GA, USA; 'Department of Pathology, Emory University School of Medicine, Atlanta, GA, USA
}

\section{Keywords}

Cornea $\cdot$ Conjunctiva $\cdot$ Tumors $\cdot$ Melanoma $\cdot$ Primary

acquired melanosis $\cdot$ Corneal extension

\begin{abstract}
Aim: The aim of this study is to describe the clinical and pathologic features of corneal primary acquired melanosis (PAM) and melanoma. Methods: We describe 3 cases in total: two cases of corneal melanomas and 1 case of corneal PAM. The eyes were processed routinely for histopathological examination. Clinical histories, treatments, and outcomes were reviewed. Results: Corneal melanomas arose from recurrence of conjunctival melanoma or conjunctival PAM at the limbus. One patient had a recurrence after excision of a limbal melanoma, another had a de novo corneal melanoma, and the last patient had corneal PAM in the setting of conjunctival PAM with atypia. All lesions were excised with adjuvant alcohol debridement and cryotherapy with no recurrence ranging from 1 week to 8 years. Conclusions: Corneal melanomas arise at the limbus from corneal PAM or conjunctival atypia. They can appear after excisional removal of a conjunctival melanoma. Surgical excision with alcohol debridement and adjuvant cryotherapy is successful.
\end{abstract}

(c) 2021 S. Karger AG, Basel

\section{Introduction}

Conjunctival melanomas comprise $2 \%$ of all ocular tumors and can grow to extend onto the cornea $[1,2]$. These lesions differ clinically from isolated corneal melanomas, tumors with no evidence of conjunctival melanoma that account for $5 \%$ of primary ocular surface melanomas [3]. There have been a number of cases of corneal melanomas reported, and several theories proposed to explain the etiology of this lesion [4-10]. The current concept is corneal melanoma arises from contiguous spread of a small, undetectable conjunctival melanoma, best explained by the term "corneally displaced conjunctival melanoma" [3-6]. This is based on the notion that healthy corneal tissue lacks melanocytes. Melanocytes can also undergo malignant change and extend into the corneal epithelium from the limbus; this could be seen with primary acquired melanosis (PAM) of the conjunctiva with atypia $[3,8]$. Further, there are cases of both pigmented and nonpigmented corneal melanomas after blast injury, raising the possibility that melanocyte displacement into the cornea may be a nidus [11]. Given the shared neural crest origin of melanocytes and peripheral nerves, it is also possible that these tumors may originate from melanocytes accompanying these nerves of the cornea [8]. 
There continues to be a debate regarding the etiology and classification of these tumors in literature. Herein, we report the clinical features of 2 cases who had corneal melanoma and a case of corneal PAM seen at our institution.

\section{Methods}

This retrospective study was granted exempt status by the Emory University Institutional Review Board and included that no further patient consents were necessary. Information was gathered and secured in compliance with the Health Insurance Portability and Accountability Act. A retrospective review of adults who were referred for evaluation of an ocular surface mass was undertaken. Patients were identified by a computerized search of billing records at the Emory Eye Center for the International Classification of Disease (ICD-9) codes for "malignant neoplasm of cornea," "conjunctival pigmentations," "conjunctival lesion," "unspecified disorder of conjunctiva," and "unspecified disorder of cornea." All patients were seen by the ocular oncology service at Emory Eye Center. Charts were reviewed for the period of January 1, 2000-July 1, 2020, inclusive. We included adults who had a corneal lesion suspicious for malignancy on clinical examination without any clinically evident limbal involvement. Charts were reviewed and the following data were extracted: age, gender, clinical findings at presentation, and clinical findings at last follow-up, management strategy, and recurrence status.

\section{Results}

The computerized search revealed 471 records. Of these, 96 had conjunctival pigmentation, with 82 of them identified as conjunctival PAM. Two patients had unilateral corneal melanoma. One patient had unilateral corneal PAM in addition to conjunctival PAM. The 2 cases of corneal melanoma were in men and the corneal PAM was in a woman. All of them were Caucasians, with an age range of 37-69. Regardless of prior surgical history, all lesions underwent excisional removal and adjuvant cryotherapy before histopathological examination.

The first case was a 69-year-old white male, who was referred to our ocular oncology service for management of a recurrent left limbal lesion. This patient underwent an incisional biopsy at a different medical center. The pathologic diagnosis at Emory Eye Center determined that this was a conjunctival melanoma arising from PAM on pathologic examination. Five years later, he returned with a left corneal lesion. An elevated nodular fleshy pink amelanotic lesion was present on the cornea between
12:00 and 3:00 with associated feeder vessels (Fig. 1a). The patient underwent an excisional biopsy of the lesion with alcohol debridement of the cornea and cryotherapy to the base and surrounding conjunctiva. Pathologic examination showed corneal melanoma (Fig. 1b) which was $0.6 \mathrm{~mm}$ in thickness. Immunohistochemical stains for SOX10 were positive in the tumor (Fig. 1c). Postoperatively, the patient was treated with mitomycin $\mathrm{C}$ (MMC) $0.05 \%$ topically 4 times a day for 1 week on, 1 week off for 4 weeks duration after insertion of a punctal plug. Nine months after surgery, the patient's vision was $20 / 20$ in the left eye and there was no evidence of recurrence (Fig. 1d). To date, there has been no recurrence, 2 years after surgery.

The second case was a 45-year-old white man, who was evaluated for a right limbal amelanotic corneal lesion at 3:00 with an associated feeder vessel (Fig. 2a). No conjunctival lesion was seen, and there was no visible conjunctival pigment. He underwent excision of the cornea lesion with alcohol debridement, and a section of adjacent conjunctival was also excised to see if there was any associated conjunctival tumor or PAM. Cryotherapy was applied at the limbus, allowing the freeze to extend onto the cornea. Histopathology demonstrated lobules and sheets of cells with pleomorphic round to oval, vesiculated nuclei, prominent nucleoli, and variable nuclear-to-cytoplasmic ratios (Fig. 2b). Immunohistochemical stains were positive for HMB45 in the lesion (Fig. 2c). There was PAM present in the limbal margin of the corneal specimen, but there was no adjacent PAM in the adjacent conjunctiva (Fig. 2d). The tumor measured $1.5 \mathrm{~mm}$ in thickness. Postoperatively, the patient was treated with MMC $0.04 \%$ topically 4 times a day for 1 week on, 1 week off for 4 weeks duration, but the patient developed contact dermatitis and the MMC was discontinued. After excision, there was no evidence of recurrence since the surgery was performed 8 years ago (Fig. 2e).

The third case was a 37-year-old white woman, who was evaluated for conjunctival pigmentation in both eyes and a lesion extending onto her left cornea. She had a flat pigmented mass extending to the cornea at 3:00 measuring $5 \times 4 \mathrm{~mm}$ on her left eye (Fig. 3a). There was brown pigmentation of the conjunctiva in both eyes, as well as a small flat pigmented mass temporal to the limbus measuring $2 \times 2 \mathrm{~mm}$ on the right eye. She underwent excision of the corneal lesion with alcohol debridement, and cryotherapy was applied at the limbus, with the freeze extending onto the cornea. Histopathology of the corneal lesion showed melanocytic proliferation in the basilar portion 

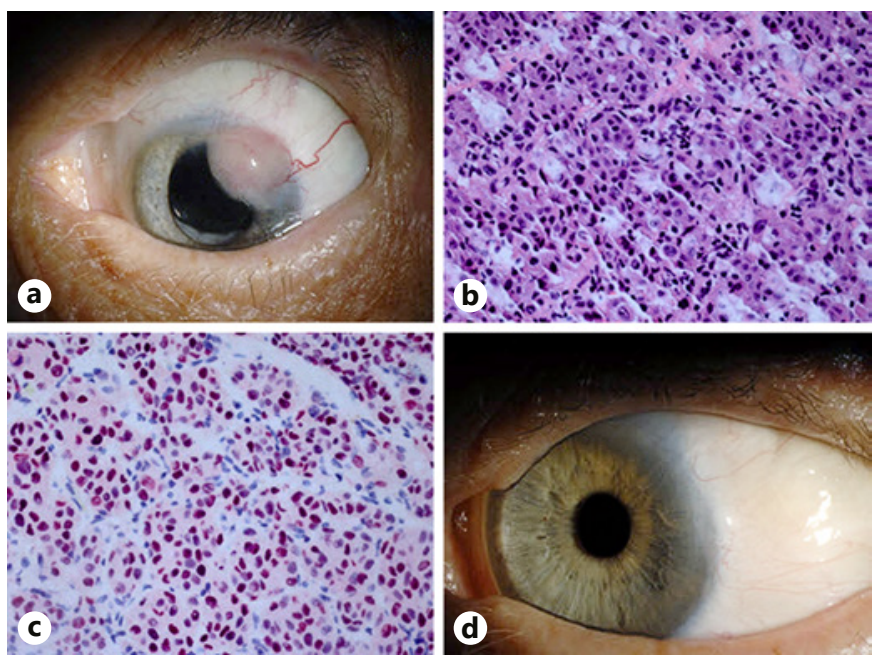

Fig. 1. Patient 1. a Clinical appearance of corneal melanoma with no visible limbal component. b The corneal and adjacent conjunctival tissue showed a tumor is composed of collections of cells with high nuclear-to-cytoplasmic ratios. c Immunohistochemical stains for SOX 10 are positive in tumor cell nuclei. d Postoperative appearance 10 months after surgery shows no residual disease (hematoxylin and eosin $\times 25$ (b); peroxidase anti-peroxidase $\times 25(\mathbf{c})$ ).

of the epithelium that form nests, consistent with PAM (Fig. 3b). The adjacent conjunctiva demonstrated junctional melanocytic proliferation, including nests and melanocytes with pleomorphic oval nuclei and variable nuclear-to-cytoplasmic ratios. These cells extended into approximately one-third of full epithelial thickness, suggestive of PAM with moderate atypia (Fig. 3c). The conjunctival lesion measured $0.5 \mathrm{~mm}$ in thickness. The patient was discharged on corticosteroid eye drops. There is no evidence of recurrence at 6-month follow-up.

\section{Discussion}

This study provides a single-center experience of corneal melanomas and corneal PAM. The first case showed a recurrence of limbal melanoma from conjunctival $\mathrm{PAM}$, and the second case presented a limbal corneal melanoma with PAM at the limbal margin of the corneal lesion. The third case demonstrated corneal PAM with adjacent conjunctival PAM, strengthening the hypothesis that these lesions originate at the limbus. Our cases also suggest that these lesions arise from conjunctival displacement of PAM, supporting the theory that they are corneally displaced conjunctival dysplasias.
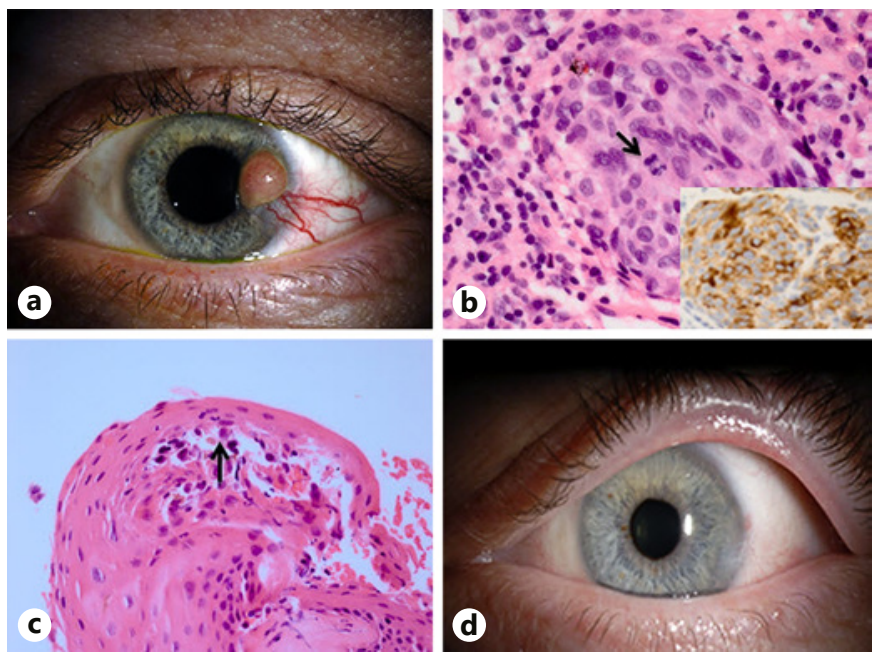

Fig. 2. Patient 2. a Clinical appearance of corneal melanoma with a clear zone between the cornea melanoma and the limbus. b The melanoma consists of collections of cells with variable nuclear-tocytoplasmic ratios. A mitotic figure is present (arrowhead). Inset Immunohistochemical stains are positive for HMB45 (brown) in tumor cell cytoplasm. c The conjunctival edge of the tumor contains a junctional proliferation of melanocytes (arrow) consistent with PAM . d Postoperative appearance after 8 years show no residual disease (hematoxylin and eosin, $\times 100(\mathbf{b})$; peroxidase antiperoxidase $\times 100$ (inset); hematoxylin and eosin, $\times 100$ (c)). PAM, primary acquired melanosis.
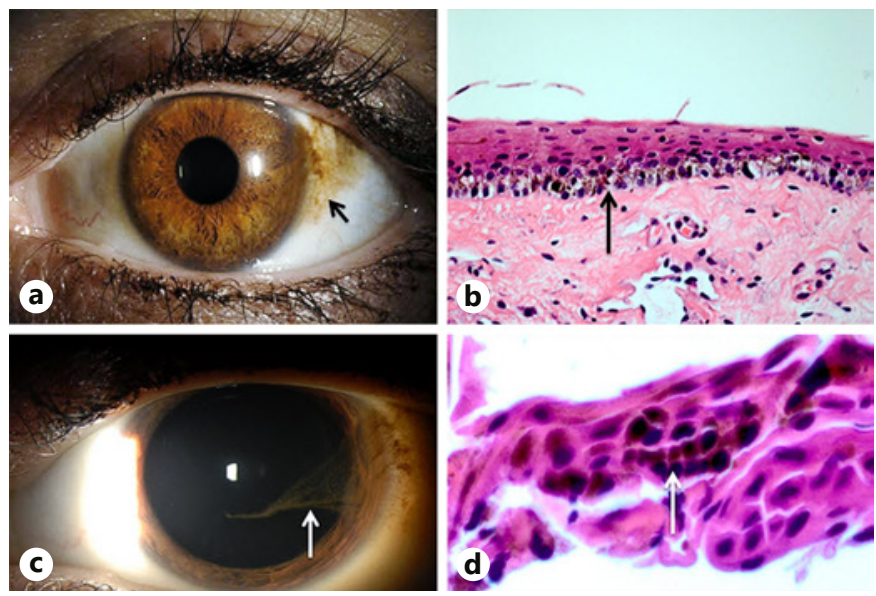

Fig. 3. Patient 3. a Clinical appearance of conjunctival PAM (arrow). $\mathbf{b}$ The conjunctiva shows proliferation of melanocytes and cells with high nuclear-to-cytoplasmic ratios (arrow), consistent with PAM with moderate atypia. c There is a whorl-like appearance to pigmentation (arrow) in the adjacent cornea. $\mathbf{d}$ The cornea demonstrates junctional growth of melanocytes consistent with PAM (hematoxylin and eosin, $\times 100$ (b); hematoxylin and eosin, $\times 100($ d)). PAM, primary acquired melanosis. 
Unlike melanomas of the conjunctiva, there have been very few reported cases of melanomas occurring at the cornea surface. Most melanomas of the conjunctiva arise in PAM, with some cases arising de novo [2, 12]. A study of 85 patients in Finland over a 34 year period showed that only 4 patients (5\%) presented with a primary invasive melanoma limited to the cornea [3]. The authors classified these tumors as corneally displaced primary malignant conjunctival melanomas including Type I, Type II, and Type III cases. In Type I, the tumor is separated from the limbus by a visible clear cornea. In Type II, the tumor parallels the cornea limbus without conjunctival invasion. In Type III, the origin is equivocal, yet the tumor epicenter is corneal and involves the limbal conjunctiva. All types are further subclassified into (A) without evidence of PAM and (B) with evidence of PAM. No patient had clinical or histopathological evidence of conjunctival tumor other than PAM.

The first patient in their study had a Type IA tumor, which was separated from the limbus by a clear cornea with no conjunctival PAM. This presentation is similar to our second case, with no conjunctival PAM observed on histopathological examination. Their second and third patients had type IB tumors with limbal corneal and conjunctival PAM similar to our first case. Their fourth case did not clinically involve the conjunctiva yet had a corneal melanoma parallel to the limbus (type IIA). Though 2 patients in the series had no appreciable PAM, the authors concluded that corneal melanomas may originate from corneal migration of conjunctival PAM, which can be amelanotic or difficult to clinically appreciate [3]. It is also possible that there was PAM sine pigmento at the limbus in our first 2 cases. This is supported by our third case, which shows concurrent corneal and conjunctival PAM and would have been classified as a IIIB if the corneal PAM had undergone malignant transformation.

There are various hypotheses as to how corneal melanomas arise. One thought is that primary conjunctiva melanoma spreads directly to the cornea. Possible melanocytes from the limbus are carried along with transient amplifying cells that migrate from limbal stem cells to the basal region of the cornea layer $[13,14]$. An alternative theory is that melanocytes move from the limbus to the adjacent corneal epithelium during injury or the effect of solar radiation [15]. Our cases support the theory that these lesions arise at the limbus and may extend to the cornea. The melanoma in our first patient arose from PAM that extended onto the cornea, and our second patient had PAM at the edge of the lesion. Finally, Case 3 showed corneal PAM adjacent to conjunctival PAM, a unique finding suggesting that the migration of PAM from the conjunctiva onto the cornea is key in the pathogenesis of these rare tumors.

Our recommended treatment for corneal melanoma is complete excision with absolute alcohol debridement of Bowman's later and adjuvant double freeze-thaw cryotherapy to the adjacent conjunctiva free margins. We recommend excising the limbal tissue for at least $2 \mathrm{~mm}$ adjacent to the conjunctival tumor with adjuvant cryotherapy. It is important to aim for excisional biopsy on the first surgery, as these lesions can recur if complete excision is not performed (Case 1). It is crucial that these lesions be referred to an ocular oncology service for management. The recurrence rate for conjunctival melanomas has been reported to be $65 \%$ at 15 years and $5 \%$ have 4 more recurrences [12]. Due to potential recurrences, we recommend long-term follow-up with serial slit lamp photography and clinical examination. In our patient group, the first case has been followed up for 2 years and the second case for 9 years, and to date, no further recurrences have occurred.

Overall, our study provides support that corneal tumors originate from conjunctival melanocytic migration. These tumors arise at the limbus and can be called "corneally displaced conjunctival melanomas." Complete excision of the lesions, alcohol debridement with preservation of Bowman's layer, and adjuvant cryotherapy result in favorable outcomes. Larger studies need to be conducted to confirm our findings and elucidate the features of these intriguing tumors.

\section{Statement of Ethics}

This retrospective study was granted exempt status by the Emory University Institutional Review Board and included that no further patient consents were necessary (February 2020). This study complied with the guidelines for human studies, and the research was conducted ethically in accordance with the World Medical Association Declaration of Helsinki.

\section{Conflict of Interest Statement}

The authors have no conflicts of interest to declare.

\section{Funding Sources}

Supported in part by a departmental grant NIH P30EY06360. 


\section{Author Contributions}

A.Y.: conception and design, data collection, data interpretation, drafting of the manuscript, final approval of version, and agree to be accountable for all aspects of the work. O.E.U.: conception and design, data collection, data interpretation, drafting of the manuscript, final approval of version, and agree to be accountable for all aspects of the work. J.R.W.: conception and design, critical revision of the manuscript, final approval of version, and agree to be accountable for all aspects of the work. H.E.G.: conception and design, critical revision of the manuscript, final approval of version, and agree to be accountable for all aspects of the work.

\section{References}

1 Wong JR, Nanjp AA, Galor A, Karp CL. Management of ocnjunctival malignant melanoma: a review and update. Expert Rev Ophthalmol. 2015;9(3):185-204.

2 Shields CL, Shields JA. Tumors of the conjunctiva and cornea. Surv Ophthalmol. 2004; 49(1):3-24

3 Tuomaala S, Aine E, Saari KM, Kivelä T. Corneally displaced malignant conjunctival melanomas. Ophthalmology. 2002;109(5):914-9.

4 Reese A. Tumors of the eye. 2nd ed. Hoeber; 1963.

5 Uçakhan-Gündüz Ö, Gündüz K, Karsloğlu MZ, Okçu-Heper A, Kanpolat A. Corneal melanoma: report of three cases. Eye Contact Lens. 2012;38(5):341-4.
6 Romaniuk W, Koziol H, Muskalski K, Dorecka M, Tarnawska D, Sabat D. A unique case of primary corneal melanoma. Jpn J Ophthalmol. 2002;46(1):114-6.

7 Davies WS, Bailey WH. Malignant melanoma of the cornea; report of a case. AMA Arch Ophthalmol. 1954;52(6):923-4.

8 Paridaens ADA, Kirkness CM, Garner A, Hungerford JL. Recurrent malignant melanoma of the corneal stroma: a case of "black cornea”. Br J Ophthalmol. 1992;76(7):444-6.

9 Schofield PB. Non-pigmented intra-epithelial melanoma of cornea. Br J Ophthalmol. 1958; 42(2):99-105.

10 Panagiotou DZ, Chranioti AA, Tzorakoleftheraki SE, Ziakas NG, Oikonomidis PK. Primary melanoma of the cornea. GMS Ophthalmol Cases. 2020;10:Doc12-5.
11 Naseri A, Char DH, Howes E, Paglen P. Amelanotic corneal melanoma after a blast injury. Am J Ophthalmol. 2001;131(2):259-60.

12 Shields CL, Shields JA, Gündüz K, Cater J, Mercado GV, Gross N, et al. Conjunctival melanoma: risk factors for recurrence, exenteration, metastasis, and death in 150 consecutive patients. Arch Ophthalmol. 2000; 118(11):1497-507.

13 Henkind P. Migration of limbal melanocytes. Nature. 1967;214(5095):1349-51.

14 Smolin G, Thoft R. The cornea: scientific foundations and clinical practice. 2nd ed.; 1987.

15 Utman SA, James JN, Ramsden KL, Baig HM Invasive malignant melanoma of the cornea after industrial injury. Int Ophthalmol. 2014; 34(2):289-91. 